\title{
Strategic Wholesale Pricing and Commonality Strategy in a Supply Chain with Quality Segmentation
}

\author{
Tiantian Xu, Tiaojun Xiao, and Chen Tian \\ School of Management Science and Engineering, Nanjing University, Nanjing 210093, China \\ Correspondence should be addressed to Tiaojun Xiao; xiaotj@nju.edu.cn
}

Received 26 September 2014; Accepted 19 March 2015

Academic Editor: Ben T. Nohara

Copyright ( 2015 Tiantian Xu et al. This is an open access article distributed under the Creative Commons Attribution License, which permits unrestricted use, distribution, and reproduction in any medium, provided the original work is properly cited.

\begin{abstract}
We develop two game models of a one-supplier and one-manufacturer supply chain to investigate the supplier's strategic wholesale pricing decision and the manufacturer's commonality strategy. The manufacturer has three commonality strategies for the highend and low-end products: common high-quality component, common low-quality component, and dedicated components. We consider both wholesale price first scenario and commonality strategy first scenario. Under the wholesale price first scenario, we identify the range of each commonality strategy and find that (i) the common low-quality component strategy is harmful to the supplier; (ii) if the quality of low-quality component and the unit production cost of high-quality component are sufficiently low, the supplier induces the common high-quality component strategy by strategically decreasing the unit wholesale price of highquality component, while if they are sufficiently high, the supplier induces the dedicated components strategy by increasing the unit wholesale price of high-quality component and decreasing that of low-quality one. Under the commonality strategy first scenario, the common low-quality component strategy may exist. By comparing the two scenarios, we find that (i) if the unit production cost of low-quality component is medium, the equilibrium outcomes under both scenarios are identical; (ii) there exists a first-mover advantage for the two players.
\end{abstract}

\section{Introduction}

In reality, consumers are heterogeneous following their valuations over product quality. To target different consumers, manufacturers provide variant products with different quality levels. For example, Lenovo offers two main series IdeaPad and ThinkPad laptops and provides six different types for each series. When designing product line, one of the important problems for managers is whether to use a common component across different products or not. In practice, common component has been widely used as a business strategy; for example, General Motors uses the same engine and platform across Lexus and Camry [1]. In 2013, Apple used Cellular and Wireless, Display, Headphones, and Connector as common components within the new products iPhone 5 s and iPhone 5 c. Some manufacturers purchase key components from the upstream suppliers and then use them to assemble different products with their own materials and production skill. When purchasing key components, the manufacturer uses dedicated components to differentiate the products or adopt a common component for a high quality-price performance. For example, in computer industry, Toshiba uses the same type of Intel's CPU to produce computers, such as L40AC05W1 and C40-Q-AT01W1, together with Toshiba's own hard disk. However, when releasing a new product T240, Lenovo offers two versions with different Intel's CPUs: I3 and I5. Product line configuration of the manufacturer affects product substitutability as well as the price competition between the products within a product line. Further, it affects the upstream supplier's wholesale price decision. In this paper, we will investigate the strategic wholesale pricing decision of the supplier.

The extant literature about common component considers the manufacturer's commonality strategy [2], where the supplier's wholesale price behavior is ignored. However, the commonality strategy affects the order quantities of key components as well as the profit of the supplier providing key components. When the manufacturer chooses a commonality strategy to maximize his profit, the supplier's benefit may be harmed. Thus, the supplier will strategically adjust her 
unit wholesale price to induce the manufacturer to choose the commonality strategy that maximizes the supplier's profit. The manufacturer's commonality strategy complicates the supplier's wholesale price decision. This paper will investigate how the supplier strategically makes the wholesale price decision when the manufacturer decides whether to use a common component.

To be specific, we develop two game models of a onesupplier and one-manufacturer supply chain to investigate the supplier's wholesale pricing strategy and the manufacturer's commonality strategy, where the manufacturer produces two products. Each product is assembled from two key components, where one is purchased from the supplier while the other is produced in-house. The manufacturer decides whether to buy the same component. If the manufacturer chooses a common component, then the manufacturer decides to choose the common high-quality component or the common low-quality one from the supplier. We derive the demand functions from the consumer's utility, which is influenced by the quality levels and prices of the products. We consider two scenarios: wholesale price first (WPF) scenario and commonality strategy first (CSF) scenario. Under WPF scenario, the supplier strategically offers a unit wholesale price to induce a commonality strategy. We identify the range of each commonality strategy and find that the common low-quality component strategy is not an equilibrium because product differentiation can improve the supplier's profit such that the supplier strategically offers a unit wholesale price to induce dedicated components strategy. Under CSF scenario, the manufacturer chooses commonality strategy before the supplier offers the unit wholesale price. We give the range of using a commonality strategy and find that the common lowquality component strategy exists when the unit production cost of the low-quality component is medium, which is inconsistent with that under WPF scenario; the supplier's profit under WPF scenario is higher than under CSF scenario while the manufacturer's profit under WPF scenario is lower.

\section{Literature Review}

This paper is closely related to the literature that examines the implication of common component in product line design problems. Whether to use common component across products $[2,3]$ and using which kind of common component [4] are two very important decisions. Bernstein et al. [5] demonstrate that the commonality decision will not affect equilibrium decisions when the component is used in the whole line, while it can influence the optimal decisions when the component is used in part of line. Common component may benefit the low-end product while harm the high-end one [6]. Using common component may be accompanied with economies of scale $[7,8]$ and design cost reduction effect $[1,9]$. Desai et al. [1] just consider one kind of design cost while Heese and Swaminathan [9] replenish their work with several cost forms. Subramanian et al. [10] study the impact of remanufacturing on the commonality strategy. There are two reviews done by Labro [11] and Fixson [12]. The above models focus on commonality issues from the manufacturer's perspective and study the trade-off between the cost reduction and cannibalization effect. However, we study the commonality strategy from the supply chain's perspective, especially the vertical interaction between the supplier and the manufacturer.

Our paper is also related to product line design problems. Within a product line, products can be vertically or horizontally different. There are models that integrate both horizontal and vertical differentiations to solve the product line design problems $[1,8,13]$. For the horizontal one, some researchers show the optimal variety level through making the trade-off between market demand and production cost [14-16]. The others consider the product line decisions in the supply chain setting to show the relationship between variety and double marginalization effect [17-19]. The vertical differentiation model is pioneered by Mussa and Rosen [20] and extended by Moorthy [21]. After that, various product line design problems of the manufacturer are considered, such as facing a downstream distributor [22], common component $[1,8]$, special development intensive products with fixed cost [23], and different production costs [24]. These models mainly assume that there are two exogenous market segments. Market segmentation can be influenced by some market tools, such as product positioning [25] and operationrelated costs [26]. We investigate when a common component should be used in a supply chain, where there are two market segments.

This paper is also related to the strategic wholesale pricing decision. Although the wholesale price is a very common decision in a supply chain model, strategic wholesale pricing decision is rarely studied. Strategic wholesale pricing means that the supplier can strategically offer a unit wholesale price to induce the downstream firm or the potential entrant supplier to choose a strategy that is beneficial to the supplier. For example, the supplier can strategically choose the unit wholesale price to deter or allow the entry of the potential entrant supplier [27]. That is, the upstream supplier can induce the downstream firm to choose a strategy through adjusting the unit wholesale price. In this paper, we investigate how the supplier offers a strategic wholesale pricing decision to induce a commonality strategy.

In summary, this paper contributes to the literature by considering the strategic wholesale pricing and commonality decisions in a one-supplier and one-manufacturer supply chain. We investigate how the supplier makes the wholesale price decision when facing a downstream manufacturer, who has three potential product line configuration strategies. We consider WPF scenario and CSF scenario. We examine the effect of game sequence on equilibrium outcome and profits by comparing the two scenarios.

\section{The Basic Model (Wholesale Price First (WPF) Scenario)}

Consider a supply chain consisting of one supplier (she) and one manufacturer (he), where the manufacturer purchases 
one of key components, denoted by $A$, from the supplier. The manufacturer produces the products with component $A$ and his own component $B$. To target different consumer segments, the manufacturer provides high-end product $(H)$ and low-end product $(L)$, We refer to product $L$ as segment $i, i=H, L$. Following Desai et al. [1], we assume that the product's quality perceived by consumers can be expressed as $q_{i}, i=H, L$, where $q_{i}$ is the product quality of segment $i$; $q_{H}>q_{L}>0$. Providing quality $q_{A i}$ for component $A$ in segment $i$ incurs a marginal $\cos c_{A i}$ to the supplier; $c_{A H}>c_{A L}$. To focus on strategic factors, we normalize the manufacturer's marginal cost for component $B$ in segment $L$ to zero. Let the manufacturer's marginal cost for component $B$ in segment $H$ be $c_{B H}>0$.

The manufacturer can choose one or two types of component $A$ to finish the production of the end products together with his own component $B$. The manufacturer has three commonality strategies: (a) common low-quality component $A, L L$; (b) common high-quality component $A, H H$; (c) two different quality levels for component $A$ to provide dedicated products (dedicated components), $L H$. For example, we use superscript " $L H$ " to represent commonality strategy $L H$. The quality of the end product depends on its configuration profile. The product quality can be perceived by consumers as $q_{i}^{L H}=\omega_{A} q_{A i}+\omega_{B} q_{B i}, i=H, L$, where $\omega_{A}$ and $\omega_{B}$ are importance weights that consumers endow to components $A$ and $B$, respectively. If the manufacturer chooses strategy $L L$, the qualities are $q_{i}^{L L}=\omega_{A} q_{A L}+\omega_{B} q_{B i}$, while quality levels can be expressed as $q_{i}^{H H}=\omega_{A} q_{A H}+\omega_{B} q_{B i}$ if the manufacturer adopts strategy $H H$. So, we have $q_{L}^{L H}=q_{L}^{L L}, q_{H}^{L H}=q_{H}^{H H}$, and $q_{H}^{H H}-$ $q_{L}^{H H}=\omega_{B}\left(q_{B H}-q_{B L}\right)=q_{H}^{L L}-q_{L}^{L L}$.

We consider a market in which consumers have heterogeneous valuations over quality. Let the quality valuation of consumer be $\theta$ and the retail price of product $i$ be $p_{i}$. The utility function of the consumer buying product $i$ is $U\left(\theta, p_{i}, q_{i}\right)=\theta q_{i}-p_{i}$. We normalize market size to one. Following Chayet et al. [25] and $\mathrm{Yu}$ [26], we assume that quality valuation $\theta$ is uniformly distributed over the interval $[0,1]$. A consumer buys a product when the consumer can achieve the maximal positive utility. The consumer buys the high-end product only when $\theta q_{H}-p_{H} \geq \theta q_{L}-p_{L}$ and $\theta q_{H}-p_{H} \geq 0$, where $\theta q_{H}-p_{H} \geq \theta q_{L}-p_{L}$ is equivalent to $\theta \geq \theta_{1}=$ $\left(p_{H}-p_{L}\right) /\left(q_{H}-q_{L}\right)$, and the consumer buys the low-end product when $\theta q_{L}-p_{L} \geq \theta q_{H}-p_{H}$ and $\theta q_{L}-p_{L} \geq 0$; explicitly, $\theta \in\left(p_{L} / q_{L}, \theta_{1}\right)$. Similar to Yu [26], we can depict Figure 1. Define $\theta_{2}=p_{L} / q_{L}(>0)$. From $\theta>\theta_{1}>\theta_{2}$, it follows that $\theta q_{H}-p_{H} \geq 0$; that is, the participation constraint of the consumer with $\theta>\theta_{1}$ is satisfied. The consumers with $\theta \in\left[\theta_{1}, 1\right]$ will buy the high-end product while the consumers with $\theta \in\left[\theta_{2}, \theta_{1}\right)$ will buy the low-end product. Further, the demand for the high-end product is $D_{H}=1-\theta_{1}$ and that for the low-end one is $D_{L}=\theta_{1}-\theta_{2} .0<\theta_{2}<\theta_{1}<1$ should be satisfied to ensure both products have positive demands.

From the definitions of $\theta_{1}$ and $\theta_{2}$, we obtain $p_{H}=q_{H} \theta_{1}+$ $q_{L}\left(\theta_{2}-\theta_{1}\right)$ and $p_{L}=\theta_{2} q_{L}$. Thus, we can substitute $\theta_{1}$ and $\theta_{2}$ for $p_{H}$ and $p_{L}$ as the decision variables. If the manufacturer chooses strategy $L H$ (i.e., using low- (high-) quality

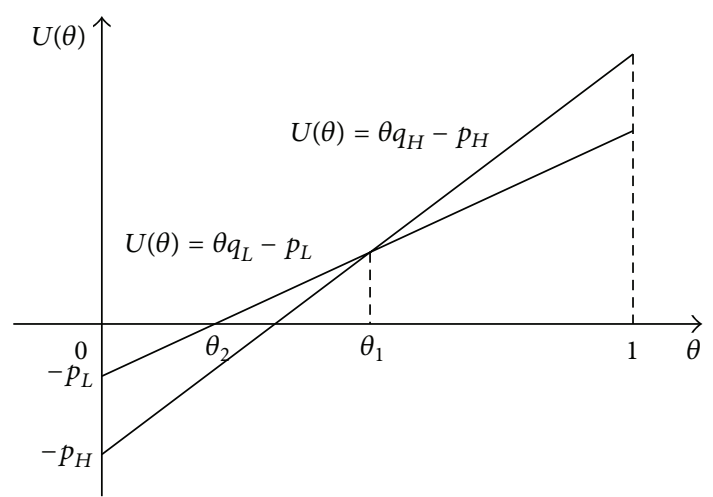

FIGURE 1: Utility of consumers versus quality valuation.

components $A$ and $B$ to produce low- (high-) quality product), the supplier's profit is

$$
\begin{aligned}
\pi_{s}^{L H} & \left(\theta_{1}, \theta_{2}, w_{A H}, w_{A L}\right) \\
& =\left(1-\theta_{1}\right)\left(w_{A H}-c_{A H}\right)+\left(\theta_{1}-\theta_{2}\right)\left(w_{A L}-c_{A L}\right),
\end{aligned}
$$

and the manufacturer's profit is

$$
\begin{aligned}
\pi_{m}^{L H} & \left(\theta_{1}, \theta_{2}, w_{A H}, w_{A L}\right) \\
= & \left(1-\theta_{1}\right)\left[q_{H}^{L H} \theta_{1}+q_{L}^{L H}\left(\theta_{2}-\theta_{1}\right)-w_{A H}-c_{B H}\right] \\
& +\left(\theta_{1}-\theta_{2}\right)\left(\theta_{2} q_{L}^{L H}-w_{A L}\right) .
\end{aligned}
$$

If the manufacturer chooses strategy $L L$, the supplier's profit is

$$
\pi_{s}^{L L}\left(\theta_{1}, \theta_{2}, w_{A L}\right)=\left(1-\theta_{2}\right)\left(w_{A L}-c_{A L}\right),
$$

where $1-\theta_{2}$ represents the total purchasing quantity for using common low-quality component $A$. The manufacturer's profit is

$$
\begin{aligned}
\pi_{m}^{L L} & \left(\theta_{1}, \theta_{2}, w_{A L}\right) \\
= & \left(1-\theta_{1}\right)\left[q_{H}^{L L} \theta_{1}+q_{L}^{L L}\left(\theta_{2}-\theta_{1}\right)-w_{A L}-c_{B H}\right] \\
& +\left(\theta_{1}-\theta_{2}\right)\left(q_{L}^{L L} \theta_{2}-w_{A L}\right),
\end{aligned}
$$

where the first-term is the manufacturer's profit from the high-end product while the second-term is the profit from the low-end one.

If the manufacturer chooses strategy $\mathrm{HH}$, the supplier's profit is

$$
\pi_{s}^{H H}\left(\theta_{1}, \theta_{2}, w_{A H}\right)=\left(1-\theta_{2}\right)\left(w_{A H}-c_{A H}\right),
$$

and the manufacturer's profit is

$$
\begin{aligned}
\pi_{m}^{H H} & \left(\theta_{1}, \theta_{2}, w_{A H}\right) \\
= & \left(1-\theta_{1}\right)\left[q_{H}^{H H} \theta_{1}+q_{L}^{H H}\left(\theta_{2}-\theta_{1}\right)-w_{A H}-c_{B H}\right] \\
& +\left(\theta_{1}-\theta_{2}\right)\left(q_{L}^{H H} \theta_{2}-w_{A H}\right) .
\end{aligned}
$$


According to the time sequences of the unit wholesale price and commonality decisions, we divide the discussions into two scenarios: WPF scenario and CSF scenario. Under WPF scenario, the manufacturer chooses commonality strategy only when he observes the unit wholesale price. Specifically, the time sequence of the game is as follows.

(i) The supplier determines the unit wholesale prices of component $A,\left(w_{A H}, w_{A L}\right)$.

(ii) Observing the unit wholesale prices, the manufacturer first chooses commonality strategy, $L L, H H$, or $L H$, and then decides the retail prices.

By using backward induction technique, we can solve the subgame perfect Nash equilibrium (SPNE).

\section{Equilibrium Analysis}

4.1. Reactions of the Manufacturer. Given the unit wholesale prices, we can obtain the retail price reaction of the manufacturer $\left(\theta_{1}\right.$ and $\left.\theta_{2}\right)$. Furthermore, we can obtain the demands and retail prices of the high-end and the low-end products, which are summarized in Proposition 1. To ensure that the demand for each product is positive, we assume that product differentiation is very large throughout this paper: $q_{H}^{L L}-q_{L}^{L L}>$ $c_{B H}$. According to the definitions of product quality, we have $q_{H}^{L H}-q_{L}^{L H}>q_{H}^{L L}-q_{L}^{L L}=q_{H}^{H H}-q_{L}^{H H}$.

Proposition 1. (i) Under strategy $L H$, the demands are

$$
\begin{gathered}
D_{H}^{L H}\left(w_{A L}, w_{A H}\right)=\frac{1}{2}-\frac{c_{B H}+\left(w_{A H}-w_{A L}\right)}{2\left(q_{H}^{L H}-q_{L}^{L H}\right)}, \\
D_{L}^{L H}\left(w_{A L}, w_{A H}\right)=\frac{c_{B H} q_{L}^{L H}+w_{A H} q_{L}^{L H}-w_{A L} q_{H}^{L H}}{2 q_{L}^{L H}\left(q_{H}^{L H}-q_{L}^{L H}\right)},
\end{gathered}
$$

and the retail prices are

$$
\begin{gathered}
p_{H}^{L H}\left(w_{A H}\right)=\frac{\left(c_{B H}+q_{H}^{L H}+w_{A H}\right)}{2} \\
p_{L}^{L H}\left(w_{A L}\right)=\frac{\left(q_{L}^{L H}+w_{A L}\right)}{2}
\end{gathered}
$$

(ii) under strategy $L L$, the demand functions are

$$
\begin{gathered}
D_{H}^{L L *}=\frac{1}{2}-\frac{c_{B H}}{2\left(q_{H}^{L L}-q_{L}^{L L}\right)}, \\
D_{L}^{L L}\left(w_{A L}\right)=\frac{c_{B H}}{2\left(q_{H}^{L L}-q_{L}^{L L}\right)}-\frac{w_{A L}}{2 q_{L}^{L L}},
\end{gathered}
$$

and the retail prices are

$$
\begin{gathered}
p_{H}^{L L}\left(w_{A L}\right)=\frac{\left(c_{B H}+q_{H}^{L L}+w_{A L}\right)}{2}, \\
p_{L}^{L L}\left(w_{A L}\right)=p_{L}^{L H}\left(w_{A L}\right) ;
\end{gathered}
$$

(iii) under strategy $\mathrm{HH}$, the demands are

$$
\begin{gathered}
D_{H}^{H H *}=D_{H}^{L L *}, \\
D_{L}^{H H}\left(w_{A H}\right)=\frac{c_{B H}}{2\left(q_{H}^{H H}-q_{L}^{H H}\right)}-\frac{w_{A H}}{2 q_{L}^{H H}},
\end{gathered}
$$

and the retail prices are

$$
\begin{gathered}
p_{H}^{H H}\left(w_{A H}\right)=p_{H}^{L H}\left(w_{A H}\right), \\
p_{L}^{H H}\left(w_{A H}\right)=\frac{\left(q_{L}^{H H}+w_{A H}\right)}{2} .
\end{gathered}
$$

Proposition 1 implies that if the manufacturer uses strategy $L H$, the demand of the high-end product increases with product differentiation (the difference between the product quality levels). When product differentiation increases, some consumers who buy the low-end product may turn to buy the high-end one. The demand of the low-end product increases with its quality level while it decreases with the quality of high-end product. That is, when the quality level of the low-end product increases, the total demand of the two products increases while the demand of the high-end one decreases. However, when the quality of the high-end product increases, the demand of the low-end one decreases because some consumers are attracted to buy the high-end product. When the unit production cost of component $B$ increases, the demand of the high-end product decreases while the low-end one increases. If the manufacturer chooses a common component, the demand of the high-end product only relates to quality difference and the unit production cost of component $B$. From $q_{H}^{L L}-q_{L}^{L L}=q_{H}^{H H}-q_{L}^{H H}$, we see that the demand of the high-end product under strategy $H H$ is equal to that under strategy $L L$; that is, $D_{H}^{H H *}=D_{H}^{L L *}$.

From Proposition 1, we know that the retail price of the high-end product increases with its own quality, the unit production cost of component $B$, and the unit wholesale price. The retail prices of the high-end product under strategies $\mathrm{HH}$ and $L H$ are identical because of $q_{H}^{L H}=q_{H}^{H H}$. If the manufacturer uses strategy $L L$, the retail price is lower due to the lower quality. Similarly, we can know that the retail prices of the low-end product under strategies $L H$ and $L L$ are identical, which is lower than that under strategy $H H$.

The demands of both high-end and low-end products should be positive. Lemma 2 gives the valid parameter settings.

Lemma 2. If the unit wholesale prices satisfy

$$
\begin{gathered}
w_{A H}-\left[\left(q_{H}^{L H}-q_{L}^{L H}\right)-c_{B H}\right]<w_{A L}<\frac{c_{B H} q_{L}^{L L}}{q_{H}^{L L}-q_{L}^{L L}}, \\
\frac{q_{H}^{L H} w_{A L}}{q_{L}^{L H}}-c_{B H}<w_{A H}<\frac{c_{B H} q_{L}^{H H}}{q_{H}^{H H}-q_{L}^{H H}},
\end{gathered}
$$

then the manufacturer will provide two end products.

Under strategy $L L$ or $H H$, when the unit wholesale price increases, the demand of the low-end one will decrease due 


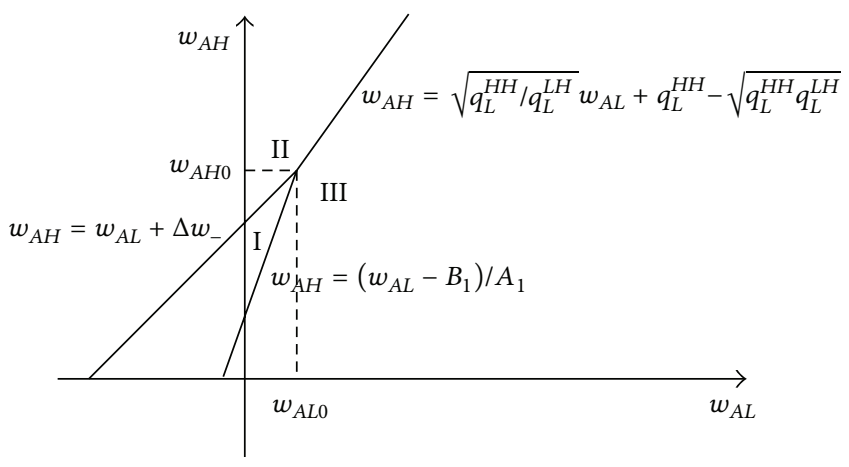

FIGURE 2: Commonality strategy versus the unit wholesale prices.

to a higher retail price. From Lemma 2, we know that the unit wholesale prices should be sufficiently low $\left(w_{A L}<\right.$ $\left.\left(c_{B H} q_{L}^{L L} /\left(q_{H}^{L L}-q_{L}^{L L}\right)\right), w_{A H}<c_{B H} q_{L}^{H H} /\left(q_{H}^{H H}-q_{L}^{H H}\right)\right)$ when the manufacturer uses common component.

By inserting the reaction functions into (2), (4), and (6), we can obtain the profit functions of the manufacturer: $\pi_{m}^{L H}\left(w_{A L}, w_{A H}\right), \quad \pi_{m}^{L L}\left(w_{A L}\right), \quad$ and $\pi_{m}^{H H}\left(w_{A H}\right)$. Lemma 3 describes the effects of the unit wholesale prices on the manufacturer's profit.

Lemma 3. (i) Under strategy LH, the manufacturer's profit is a convex and decreasing function of the unit wholesale price in each segment; (ii) under strategy LL or HH, the manufacturer's profit is a convex and decreasing function of the unit wholesale price.

Lemma 3 implies that, within the feasible region given by Lemma 2, the decreasing speed of the manufacturer's profit in the unit wholesale price becomes faster.

The manufacturer determines the commonality strategy based on the profits under different conditions. Define $F_{1}=\pi_{m}^{L H}\left(w_{A L}, w_{A H}\right)-\pi_{m}^{L L}\left(w_{A L}\right), F_{2}=\pi_{m}^{L H}\left(w_{A L}, w_{A H}\right)-$ $\pi_{m}^{H H}\left(w_{A H}\right)$, and $F_{3}=\pi_{m}^{H H}\left(w_{A H}\right)-\pi_{m}^{L L}\left(w_{A L}\right)$. The manufacturer will use strategy $L H$ if $F_{1} \geq 0$ and $F_{2} \geq 0$, the manufacturer would like to use strategy $\mathrm{HH}$ if $\mathrm{F}_{2}<0$ and $F_{3} \geq 0$, and the manufacturer would like to use strategy $L L$ if $F_{1}<0$ and $F_{3}<0$.

Proposition 4 summarizes the commonality strategy of the manufacturer given the unit wholesale prices, $w_{A L}, w_{A H}$; also see Figure 2.

Proposition 4. If $\left(w_{A L}, w_{A H}\right) \in I$, the manufacturer uses strategy LH; if $\left(w_{A L}, w_{A H}\right) \in I I$, the manufacturer will use strategy LL, while the manufacturer uses strategy $\mathrm{HH}$ if $\left(w_{A L}, w_{A H}\right) \in I I I$, where regions

$$
\begin{gathered}
I=\left\{\left(w_{A L}, w_{A H}\right) \mid w_{A L}+\Delta w_{-} \geq w_{A H} \geq \frac{\left(w_{A L}-B_{1}\right)}{A_{1}}\right\}, \\
I I=\left\{\left(w_{A L}, w_{A H}\right) \mid w_{A H}>\max \left\{w_{A L}+\Delta w_{-}, w_{A H-}\right\}\right\}, \\
I I I=\left\{\left(w_{A L}, w_{A H}\right) \mid w_{A H}<\min \left\{\frac{\left(w_{A L}-B_{1}\right)}{A_{1}}, w_{A H-}\right\}\right\},
\end{gathered}
$$

$$
\begin{gathered}
w_{A H-}=\sqrt{\frac{q_{L}^{H H}}{q_{L}^{L H}}} w_{A L}+q_{L}^{H H}-\sqrt{q_{L}^{H H} q_{L}^{L H}}, \\
A_{1}=\frac{q_{L}^{H H} q_{L}^{L H}+\sqrt{\left(q_{H}^{L H}-q_{L}^{H H}\right)\left(q_{H}^{L H}-q_{L}^{L H}\right) q_{L}^{H H} q_{L}^{L H}}}{q_{L}^{H H} q_{H}^{L H}}, \\
B_{1} \frac{q_{L}^{L H}\left(q_{H}^{L H}-q_{L}^{H H}\right)-\sqrt{\left(q_{H}^{L H}-q_{L}^{H H}\right)\left(q_{H}^{L H}-q_{L}^{L H}\right) q_{L}^{H H} q_{L}^{L H}}}{q_{H}^{L H}\left(q_{H}^{L H}-q_{L}^{H H}\right)} \\
\cdot c_{B H}, \quad\left(\left(q_{H}^{L H}-q_{L}^{L H}-c_{B H}\right)\left(q_{H}^{L L}-q_{L}^{L H}\right)\right. \\
\Delta w_{-}=\left(q_{H}^{L L}-q_{L}^{L H}-c_{B H}\right) \\
\left.\cdot \sqrt{\left(q_{H}^{L H}-q_{L}^{L H}\right)\left(q_{H}^{L L}-q_{L}^{L H}\right)}\right) \\
\left(q_{H}^{L L}-q_{L}^{L H}\right)^{-1} \cdot
\end{gathered}
$$

Proposition 4 not only answers how the manufacturer reacts to the unit wholesale prices, but also shows guidance about how the supplier should set her optimal unit wholesale price. Figure 2 shows that thresholds $w_{A L 0}$ and $w_{A H 0}$ are important values, and the manufacturer uses strategy $L H$ only when both unit wholesale prices are lower than these thresholds. From Lemma 3, we know that a high unit wholesale price of the high-quality component $A$ will lead to a low profit for the manufacturer under strategy $L H$ while it does not change the profit of the manufacturer using strategy $L L$. So the supplier can set a sufficiently high unit wholesale price $w_{A H}$ to induce the manufacturer to only purchase the lowquality component $A$. Similarly, when the supplier offers a sufficiently high unit wholesale price $w_{A L}$, the manufacturer will use strategy $H H$. When the unit wholesale price is high enough $\left(w_{A L}>w_{A L 0}\right.$ or $\left.w_{A H}>w_{A H 0}\right)$, the manufacturer gets a higher profit from using a common component.

From Proof of Proposition 4, we know that the slope of the line $w_{A H}=\left(w_{A L}-B_{1}\right) / A_{1}$ is the largest and that of the line $w_{A H}=w_{A L}+\Delta w_{-}$is the smallest.

4.2. The Unit Wholesale Prices of the Supplier. The manufacturer's commonality strategy depends on the unit wholesale prices. In this subsection, we focus on the unit wholesale price decisions. Considering the reaction functions of the manufacturer, the profit functions in different regions $(I, I I$, and $I I I)$ can be expressed as follows:

$$
\begin{aligned}
\pi_{s}^{I}\left(w_{A L}, w_{A H}\right) \\
=\left\{q_{L}^{L H}\left(w_{A H}-c_{A H}\right)\left(q_{H}^{L H}-q_{L}^{L H}-c_{B H}-w_{A H}+w_{A L}\right)\right. \\
\left.+\left(w_{A L}-c_{A L}\right)\left[\left(c_{B H}+w_{A H}\right) q_{L}^{L H}-w_{A L} q_{H}^{L H}\right]\right\} \\
\cdot\left[2 q_{L}^{L H}\left(q_{H}^{L H}-q_{L}^{L H}\right)\right]^{-1}
\end{aligned}
$$




$$
\begin{aligned}
\pi_{s}^{I I}\left(w_{A L}\right) & =\frac{\left[-w_{A L}^{2}+\left(c_{A L}+q_{L}^{L L}\right) w_{A L}-c_{A L} q_{L}^{L L}\right]}{\left(2 q_{L}^{L L}\right)}, \\
\pi_{s}^{I I I}\left(w_{A H}\right) & =\frac{\left[-w_{A H}^{2}+\left(c_{A H}+q_{L}^{H H}\right) w_{A H}-c_{A H} q_{L}^{H H}\right]}{\left(2 q_{L}^{H H}\right)} .
\end{aligned}
$$

The supplier will maximize her profit across all feasible regions. From (15)-(17), we can derive the following.

Proposition 5. (i) The supplier sells two kinds of component $A$ to the manufacturer if

$$
\pi_{s}^{I}\left(w_{A L}^{I *}, w_{A H}^{I *}\right) \geq \max \left\{\pi_{s}^{I I}\left(w_{A L}^{I I *}\right), \pi_{s}^{I I I}\left(w_{A H}^{I I I *}\right)\right\}
$$

with the SPNE unit wholesale prices

$$
\left(w_{A L}^{I *}, w_{A H}^{I *}\right)= \begin{cases}\left(w_{A L 1}^{I}, w_{A H 1}^{I}\right), & \text { if }\left(w_{A L 1}^{I}, w_{A H 1}^{I}\right) \in I, \\ \left(w_{A L 2}^{I}, w_{A H 2}^{I}\right), & \text { if } w_{A L 1}^{I}+\Delta w_{-} \geq w_{A H 1}^{I}, \\ & w_{A H 1}^{I}<\frac{\left(w_{A L 1}^{I}-B_{1}\right)}{A_{1}}, \\ & w_{A H 2}^{I}<w_{A H 0}, \\ \left(w_{A L 3}^{I}, w_{A H 3}^{I}\right), & \text { if } w_{A L 1}^{I}+\Delta w_{-}<w_{A H 1}^{I}, \\ & w_{A H 1}^{I} \geq \frac{\left(w_{A L 1}^{I}-B_{1}\right)}{A_{1}}, \\ & w_{A L 3}^{I}<w_{A L 0} \\ & \text { otherwise, }\end{cases}
$$

where

$$
\begin{gathered}
w_{A L 1}^{I}=w_{A L 3}^{I}=\frac{\left(c_{A L}+q_{L}^{L H}\right)}{2}, \\
w_{A H 1}^{I}=\frac{\left(q_{H}^{L H}+c_{A H}-c_{B H}\right)}{2}, \\
w_{A H 3}^{I}=w_{A L 3}^{I}+\Delta w_{-}, \\
w_{A L 0}=\frac{\left(B_{1}+A_{1} \Delta w_{-}\right)}{\left(1-A_{1}\right)}, \\
w_{A H 0}=\frac{\left(\Delta w_{-}+B_{1}\right)}{\left(1-A_{1}\right)}, \\
w_{A L 2}^{I}=A_{1} w_{A H 2}^{I}+B_{1}, \\
w_{A H 2}^{I} \quad ; \quad\left(q_{L}^{L H}\left(2 B_{1}+c_{A H}-c_{A L}-c_{B H}+q_{H}^{L H}-q_{L}^{L H}\right)\right. \\
\left.+A_{1}\left(-2 B_{1} q_{H}^{L H}+c_{A L} q_{H}^{L H}-c_{A H} q_{L}^{L H}+c_{B H} q_{L}^{L H}\right)\right) \\
\cdot\left(2\left(A_{1}^{2} q_{H}^{L H}+q_{L}^{L H}-2 A_{1} q_{L}^{L H}\right)\right)^{-1}
\end{gathered}
$$

(ii) if $\pi_{s}^{I I}\left(w_{A L}^{I I *}\right) \geq \max \left\{\pi_{s}^{I}\left(w_{A L}^{I *}, w_{A H}^{I *}\right), \pi_{s}^{I I I}\left(w_{A H}^{I I *}\right)\right\}$, the supplier only offers low-quality component $A$ with the SPNE unit wholesale price $w_{A L}^{I I *}=\left(c_{A L}+q_{L}^{L L}\right) / 2$; the unit wholesale price of the high-quality one would be any value that satisfies $w_{A H}^{I I *}>$ $\max \left\{w_{A L}^{I I *}+\Delta w_{-}, \sqrt{q_{L}^{H H} / q_{L}^{L H}} w_{A L}^{I I *}+\left(q_{L}^{H H}-\sqrt{q_{L}^{H H} q_{L}^{L H}}\right)\right\} ;(i i i)$ if $\pi_{s}^{I I I}\left(w_{A H}^{I I *}\right) \geq \max \left\{\pi_{s}^{I}\left(w_{A L}^{I *}, w_{A H}^{I *}\right), \pi_{s}^{I I}\left(w_{A L}^{I I *}\right)\right\}$, the supplier only offers high-quality component $A$ with the SPNE unit wholesale price $w_{A H}^{I I *}=\left(c_{A H}+q_{L}^{H H}\right) / 2$; the unit wholesale price of the low-quality one should satisfy $w_{A L}^{I I I}>$ $\max \left\{\sqrt{q_{L}^{L H} / q_{L}^{H H}} w_{A H}^{I I I *}-\left(\sqrt{q_{L}^{L H} q_{L}^{H H}}-q_{L}^{L H}\right), B_{1}+A_{1} w_{A H}^{I I I *}\right\}$.

From Proposition 5, we know that if the supplier provides two quality levels of component $A$, she determines the unit wholesale prices $\left(w_{A L 1}^{I}, w_{A H 1}^{I}\right)$ if $\left(w_{A L 1}^{I}, w_{A H 1}^{I}\right) \in I$. If the supplier can get a higher profit from only providing one component, she will try to induce the manufacturer to use a common component. The unit wholesale price of a common component increases with its unit production cost and the quality level of the low-end product. If the quality of component $B$ increases, the manufacturer can get a higher unit profit, so the supplier can increase the unit wholesale price to gain a larger unit profit.

We compare the equilibrium outcomes when the manufacturer uses common components, which is summarized in Corollary 6.

Corollary 6. (i) $w_{A H}^{I I I *}>w_{A L}^{I I *}$; (ii) $D_{L}^{H H *}+D_{H}^{H H *}<D_{L}^{L L *}+$ $D_{H}^{L L *}$ if $c_{A H} / c_{A L}>q_{L}^{H H} / q_{L}^{L L}$; (iii) the supplier can gain a higher profit under strategy $H H$ than under strategy LL only if $q_{L}^{L L}\left(q_{L}^{H H}-c_{A H}\right)^{2} \geq q_{L}^{H H}\left(q_{L}^{L L}-c_{A L}\right)^{2}$.

From Corollary 6, we know that the unit wholesale price for the common high-quality component is higher than that for the common low-quality one. When using a common component, the demands of high-end product under strategies $L L$ and $H H$ are identical such that the total demands depend on the low-end one. The retail price of the lowend product increases with the unit production cost of the low-quality component $A$ such that more consumers do not buy any product. On the other hand, a higher product quality for the low-end product attracts more consumers. As a result, the total demand under strategy $L L$ is higher than that under strategy $H H$ if the relative unit production cost of low-quality component $A$ is sufficiently low.

Since the optimal wholesale prices of the supplier under dedicated components are complex, we illustrate the monotonicity of the unit wholesale prices by employing numerical examples. Figures 3-7 show how the quality and the unit production costs may affect the optimal wholesale prices and the commonality strategy. Here the default values of the parameters are used as: $c_{A L}=1.5, c_{A H}=2.5, c_{B H}=2, q_{A L}=$ $20, q_{A H}=25, q_{B L}=12, q_{B H}=18, \omega_{A}=0.6$, and $\omega_{B}=0.4$.

From Figures 3 and 4, we know that the supplier's profit under strategy $L H$ is higher than that under strategy $L L$, which implies that the supplier has no incentive to offer a unit wholesale price to induce strategy $L L$. Intuitively, the lowquality component $A$ has a lower cost, which is beneficial to 


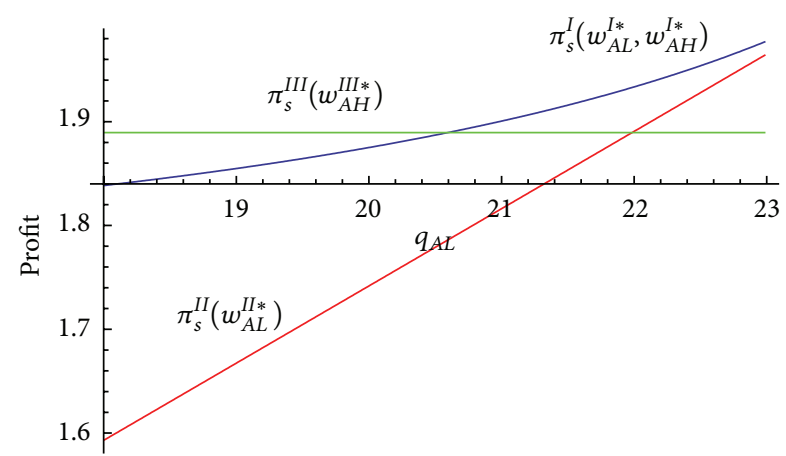

FIGURE 3: The supplier's profit versus the quality of component $A$ in segment $L$.

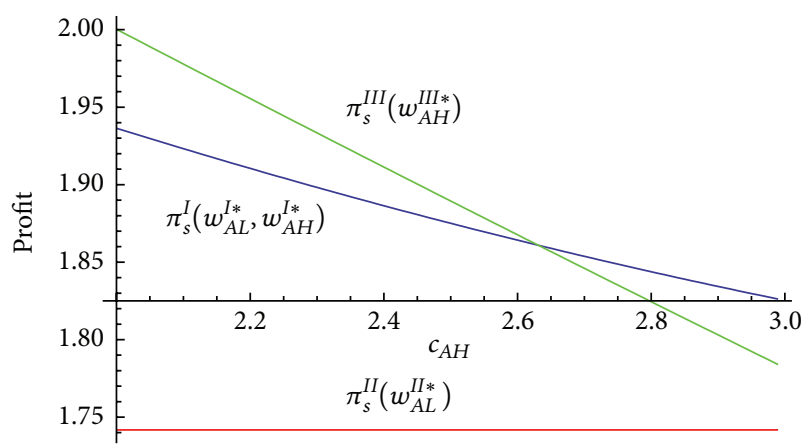

FIGURE 4: The supplier's profit versus the unit production cost of high-quality component $A$.

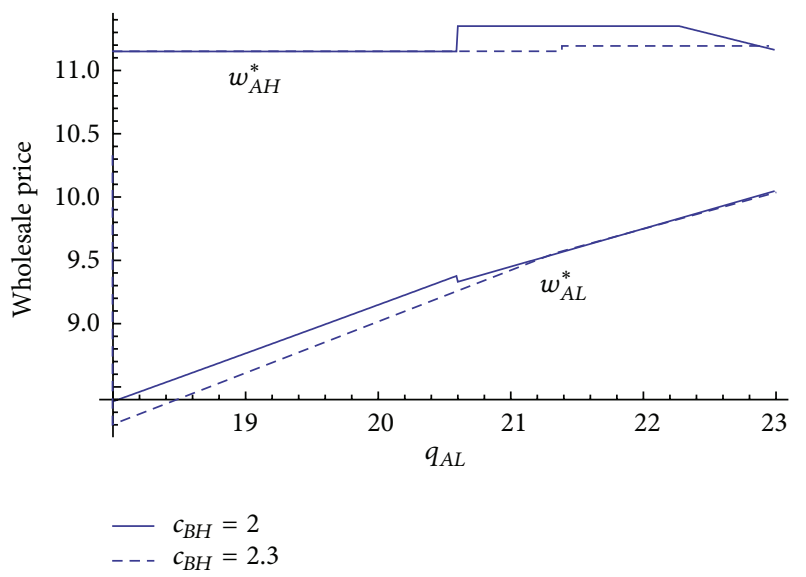

FIGURE 5: The unit wholesale prices versus $q_{A L}$ and $c_{B H}$.

the supplier. On the other hand, the quality of the high-end product under strategy $L L$ is lower than that under strategy $L H$, which is harmful to the supplier due to a lower market demand. Moreover, using strategy $L L$ decreases product differentiation, which increases the price competition. As a result, relative to strategy $L H$, the negative effect of using strategy $L L$ on the supplier outweighs its positive effect. The supplier will encourage the manufacturer to use strategy $\mathrm{LH}$ or $\mathrm{HH}$.

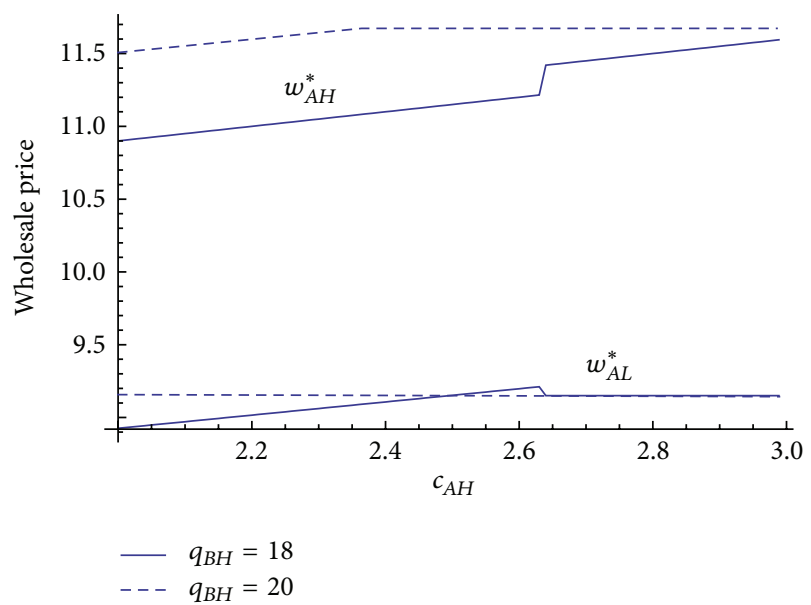

FIgURE 6: The unit wholesale prices versus $q_{B H}$ and $c_{A H}$.

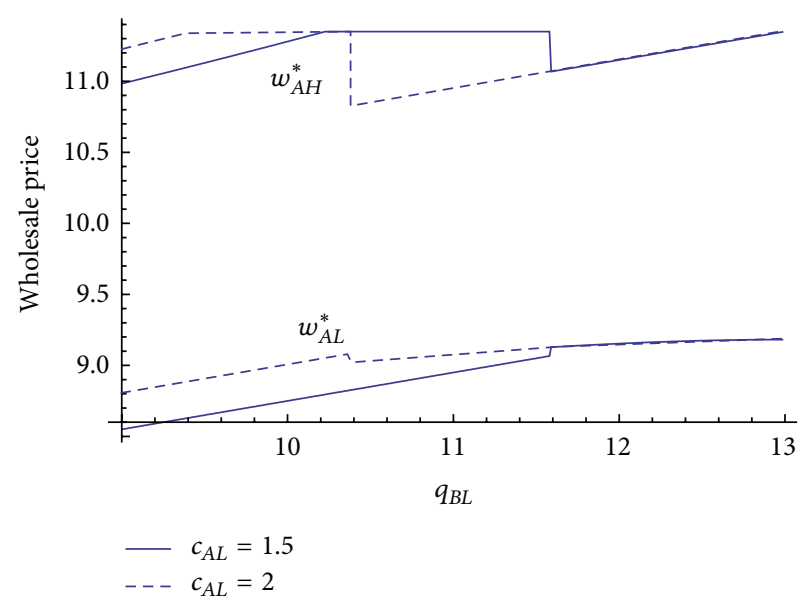

Figure 7: The unit wholesale prices versus $q_{B L}$ and $c_{A L}$.

Figure 3 implies that the supplier will induce strategy $\mathrm{HH}$ if the quality of the low-quality component $A$ is sufficiently low; otherwise, the supplier will induce strategy $L H$. Intuitively, under strategy $L H$, a higher quality of component $A$ in segment $L$ increases the supplier's profit because it reduces the quality disadvantage. Moreover, product differentiation under strategy $L H$ is larger than under strategy $H H$. As a result, the supplier's profit under strategy $L H$ is higher if the quality of component $A$ in segment $L$ is sufficiently high.

Figure 4 implies that the supplier has an incentive to induce strategy $\mathrm{HH}$ if the unit production cost of component $A$ in segment $H$ is sufficiently low. Intuitively, the negative effect of the unit production $\operatorname{cost} c_{A H}$ on the supplier under strategy $H H$ is larger than that under strategy $L H$ such that the supplier can obtain a higher profit under strategy $\mathrm{HH}$ if it is very small. Moreover, as pointed out earlier, the supplier prefers strategy $L H$ to strategy $L L$.

Figures 5-7 illustrate the impacts of the quality and unit production costs on the optimal wholesale prices. In Figure 5, the first interval matches strategy $\mathrm{HH}$ while the following one relates to strategy $L H$. Figure 5 implies that when the quality 
of component $A$ in segment $L$ is sufficiently high, the supplier will strategically increase the unit wholesale price of component $A$ in segment $H$ and decreases that in segment $L$, which induces the manufacturer to choose strategy $L H$. In addition, we find that the unit wholesale price does not increase with the unit production cost of component $B$.

In Figure 6, the two intervals match strategies $H H$ and $L H$, respectively. The supplier will be reluctant to induce the manufacturer to use $\mathrm{HH}$ when the unit production cost of component $A$ in segment $H$ is sufficiently high. When the unit production cost increases, the supplier charges a higher wholesale price for high-quality component $A$. In order to encourage the manufacturer to use strategy $H H$, the supplier also raises the unit wholesale price of the low-quality component.

The first interval relates to strategy $L H$ while the second one corresponds to strategy $H H$ in Figure 7. When the quality of component $B$ in segment $L$ is high enough, the supplier will induce the manufacturer to use strategy $\mathrm{HH}$; the supplier strategically decreases the unit wholesale price for the high-quality component $A$ to induce strategy $\mathrm{HH}$; however, whether to increase the unit wholesale price for the lowquality component $A$ depends on its unit production cost.

\section{Equilibrium Outcome under Commonality Strategy First (CSF) Scenario}

Under CSF scenario, the supplier sets the unit wholesale prices after the product line configuration is designed [28]. Specifically, the time sequence of the game is as follows.

(i) The manufacturer chooses commonality strategy: $H H, L L$, or $L H$.

(ii) Observing commonality strategy, the supplier determines the unit wholesale prices.

(iii) Observing the unit wholesale price, the manufacturer sets the retail prices.

Similar to Proposition 5, we can show that the SPNE decisions are the same as those under WPF scenario if the manufacturer uses a common component $A$; that is, the unit wholesale price under strategy $L L$ is $w_{A L}^{I I *}$ and that under strategy $H H$ is $w_{A H}^{I I I *}$. Under strategy $L H$, the unit wholesale prices are $\left(w_{A L 1}^{I}, w_{A H 1}^{I}\right)$. Note that if the manufacturer uses strategy $L L$, the supplier charges the same wholesale price as that under strategy $L H$; if the manufacturer uses strategy $H H$, he will pay more for the high-quality component $A$.

Corollary 7 gives out the conditions under which the equilibrium decisions under WPF and CSF scenarios are identical.

Corollary 7. If $c_{A L} \in\left[q_{H}^{L H}-q_{L}^{L H}-c_{B H}-2 \Delta w_{-}+c_{A H},\left(q_{H}^{L H}+\right.\right.$ $\left.\left.c_{A H}-c_{B H}\right) A_{1}+2 B_{1}-q_{L}^{L H}\right]$, product line design and pricing decisions under WPF and CSF scenarios are identical.

Corollary 7 implies that the time sequence of decisions does not affect the equilibrium decisions if the unit production cost for the low-quality component $A$ is medium. The main cause is that, within the range, the supplier can optimize her wholesale price under WPF scenario because the manufacturer has an incentive to choose strategy $L H$. Thus, the time sequence of decisions does not affect the pricing decisions under strategy $L H$. Moreover, we know that the time sequence does not change the pricing decisions under the other strategies. As a consequence, the pricing decisions and commonality strategies under two scenarios are identical. However, if the unit production $\operatorname{cost} c_{A L}$ is either too high or low, the time sequence affects the pricing decisions as well as commonality strategy.

Under CSF scenario, the manufacturer's profits are

$$
\begin{aligned}
& \pi_{m}^{L L * *} \\
& =\left(q_{L}^{L L}\left[4\left(q_{H}^{L L}-c_{B H}\right)^{2}+q_{L}^{L L}\left(8 c_{B H}-7 q_{H}^{L L}+3 q_{L}^{L L}\right)\right]\right. \\
& \left.-c_{A L}\left(2 q_{L}^{L L}-c_{A L}\right)\left(q_{H}^{L L}-q_{L}^{L L}\right)\right) \\
& \cdot\left(16 q_{L}^{L L}\left(q_{H}^{L L}-q_{L}^{L L}\right)\right)^{-1} \\
& \pi_{m}^{H H * *} \\
& =\left(q_{L}^{H H}\left[4 c_{B H}^{2}+\left(q_{H}^{H H}-q_{L}^{H H}\right)\left(4 q_{H}^{H H}-8 c_{B H}-3 q_{L}^{H H}\right)\right]\right. \\
& \left.-c_{A H}\left(2 q_{L}^{H H}-c_{A H}\right)\left(q_{H}^{H H}-q_{L}^{H H}\right)\right) \\
& \cdot\left(16 q_{L}^{H H}\left(q_{H}^{H H}-q_{L}^{H H}\right)\right)^{-1}, \\
& \pi_{m}^{L H * *} \\
& =\left(q _ { L } ^ { L H } \left[\left(q_{H}^{L H}-c_{B H}\right)^{2}+q_{L}^{L H}\left(2 c_{A H}+2 c_{B H}-q_{H}^{L H}\right)\right.\right. \\
& \left.+c_{A H}\left(c_{A H}+2 c_{B H}-2 q_{H}^{L H}\right)\right] \\
& \left.-c_{A L}\left(2 c_{A H} q_{L}^{L H}+2 c_{B H} q_{L}^{L H}-c_{A L} q_{H}^{L H}\right)\right) \\
& \cdot\left(16 q_{L}^{L H}\left(q_{H}^{L H}-q_{L}^{L H}\right)\right)^{-1} .
\end{aligned}
$$

Here the superscript “**” represents CSF scenario.

Define $\Delta \pi_{m 1}=\pi_{m}^{L L * *}-\pi_{m}^{L H * *}, \Delta \pi_{m 2}=\pi_{m}^{H H * *}-\pi_{m}^{L H * *}$, and $\Delta \pi_{m 3}=\pi_{m}^{H H * *}-\pi_{m}^{L L * *}$. When both $\Delta \pi_{m 1}$ and $\Delta \pi_{m 2}$ are negative, the manufacturer will use strategy $L H$. Proposition 8 summarizes the commonality strategy.

Proposition 8. Under CSF scenario, we have the following. (i) The manufacturer chooses strategy $L L$ for $c_{A L} \in$ $\left[c_{A L 1-}, \min \left\{c_{A L 1+}, c_{A L 3}\right\}\right]$. (ii) The manufacturer chooses strategy $H H$ if $c_{A L 2 \pm}$ exist and $c_{A L} \in\left[\max \left\{c_{A L 2-}, c_{A L 3}\right\}, c_{A L 2+}\right]$. (iii) Otherwise, the manufacturer chooses strategy $L H$, where

$$
\begin{aligned}
c_{A L 1 \pm}= & c_{A H}+c_{B H}-q_{H}^{H H}+q_{L}^{L L} \\
& \pm 2\left(q_{H}^{L L}-q_{L}^{L L}-c_{B H}\right) \sqrt{\frac{\left(q_{H}^{H H}-q_{L}^{L L}\right)}{\left(q_{H}^{L L}-q_{L}^{L L}\right)}},
\end{aligned}
$$




$$
\begin{gathered}
c_{A L 2 \pm}=\frac{B_{2} \pm \sqrt{B_{2}^{2}+4 A_{2} q_{H}^{H H} q_{L}^{H H}\left(q_{H}^{H H}-q_{L}^{H H}\right)}}{2 q_{H}^{H H} q_{L}^{H H}\left(q_{H}^{H H}-q_{L}^{H H}\right)}, \\
c_{A L 3}=q_{L}^{L L}-\left(q_{L}^{H H}-c_{A H}\right) \sqrt{\frac{q_{L}^{L L}}{q_{L}^{H H}}}, \\
A_{2}=q_{L}^{L L}\left\{q _ { L } ^ { H H } \left[c_{B H}^{2}\left(3 q_{H}^{H H}+q_{L}^{H H}-4 q_{L}^{L L}\right)\right.\right. \\
-6 c_{B H}\left(q_{H}^{H H}-q_{L}^{H H}\right)\left(q_{H}^{H H}-q_{L}^{L L}\right) \\
\left.+3\left(q_{H}^{H H}-q_{L}^{H H}\right)^{2}\left(q_{H}^{H H}-q_{L}^{L L}\right)\right] \\
-2 c_{A H} c_{B H} q_{L}^{H H}\left(q_{H}^{H H}-q_{L}^{H H}\right) \\
\left.+c_{A H}^{2}\left(q_{H}^{H H}-q_{L}^{H H}\right)\left(q_{H}^{H H}-q_{L}^{H H}-q_{L}^{L L}\right)\right\} \\
B_{2}=2 q_{L}^{H H} q_{L}^{L L}\left(c_{A H}+c_{B H}\right)\left(q_{H}^{H H}-q_{L}^{H H}\right) .
\end{gathered}
$$

Proposition 8 identifies the conditions under which the manufacturer chooses a commonality strategy. From Proposition 8, we can see that strategy $L L$ is possible under CSF scenario. This result is inconsistent with that under WPF scenario in a statistical sense (see Figures 3 and 4), which implies that the time sequence of decisions affects the existence of strategy $L L$. Specifically, strategy $L L$ is more prevalent under CSF scenario.

To better understand the effects of the time sequence of decisions on the players' profits, we depict Figures 8 and 9, where the default values are the same as in Figures 3-7.

From Figures 8 and 9, we see that the supplier's profit under WPF scenario is higher than that under CSF scenario; however, the manufacturer's profit under CSF scenario is higher. That is, there exists a first-mover advantage for the two players. Specifically, under WPF scenario, the supplier can strategically offer a unit wholesale price to induce the commonality strategy that maximizes her profit. Under CSF scenario, the manufacturer can use the retail price (so order quantity) to avoid the supplier's holdup behavior and choose the commonality strategy to maximize his profit. We examine the other default values of parameters and find that the main insight is unchanged.

\section{Conclusions}

Most papers on commonality strategy in product line design only consider manufacturer level. In reality, the supplier may take the manufacturer's behavior into consideration to decide the unit wholesale price. The vertical interaction between the supplier and the manufacturer plays an important role in making commonality strategy for the manufacturer. In this paper, we develop two game models to investigate how the vertical interaction affects commonality strategy and study how the supplier strategically decides the unit wholesale

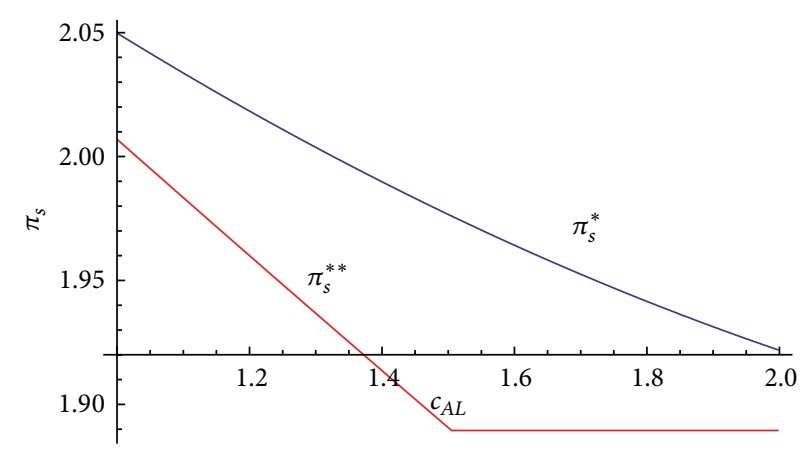

FIGURE 8: The supplier's profit versus the unit production cost of the low-quality component $A$.

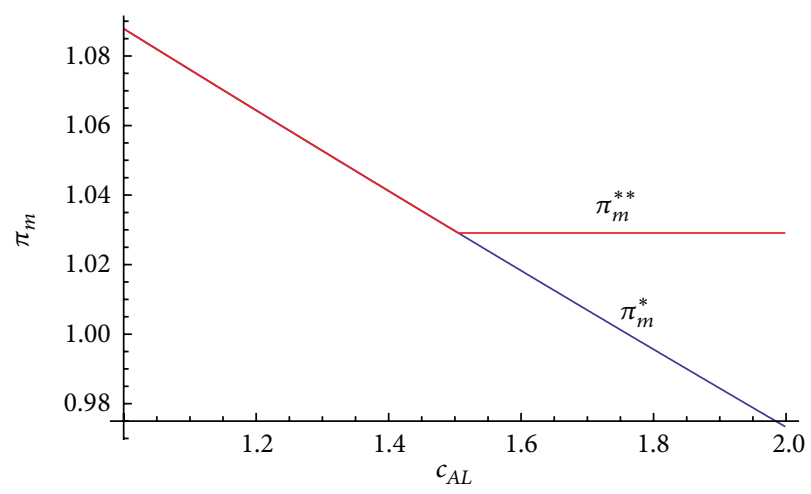

FIGURE 9: The manufacturer's profit versus the unit production cost of low-quality component $A$.

price. Here the manufacturer provides low-end product and high-end product following quality segmentation.

Under WPF scenario, the supplier first decides the unit wholesale price, and then the manufacturer determines commonality strategy. We explore how the supplier strategically offers a unit wholesale price to induce the manufacturer to choose the optimal commonality strategy for the supplier. We give the wholesale prices range of each commonality strategy and find that (i) the total demand of using common low-quality component $A$ may be higher than that of using common high-quality component $A$; (ii) the supplier can achieve a higher profit under strategy $L H$ than under strategy $L L$ because the price competition is weaker; (iii) commonality equilibrium is either strategy $L H$ or strategy $H H$; (iv) when the quality of component $A$ in segment $L$ and the unit production cost of the high-quality component $A$ are sufficiently low, the supplier will strategically decrease the unit wholesale price of the high-quality component $A$ and increase that of the low-quality one to induce strategy $\mathrm{HH}$.

Under CSF scenario, the manufacturer first decides commonality strategy, and then the supplier offers the unit wholesale price. Here the commonality strategy is regarded as a strategy tool of the manufacturer. We give the unit production cost range of each commonality strategy and find that when the unit production cost of the low-quality component $A$ is medium, the equilibrium outcome is the same as that under WPF scenario. In addition, we find that a 
first-mover advantage exists for the two players; that is, the supplier prefers WPF scenario while the manufacturer prefers CSF scenario.

This paper does not consider the effect of commonality on marginal cost. Greater commonality can reduce marginal cost [29]. One can extend to investigate how the cost reduction effect of commonality affects the commonality strategy in a supply chain. We assume that the manufacturer purchases two types of component $A$ from the same supplier. Sometimes, high-quality and low-quality components are provided by different suppliers, where the suppliers play a price game. In this case, the commonality strategy may be interesting.

\section{Appendix}

Proof of Proposition 1. The Hessian matrix of $\pi_{m}^{L H}\left(\theta_{1}, \theta_{2}\right.$, $\left.w_{A H}, w_{A L}\right)$ over $\left(\theta_{1}, \theta_{2}\right)$ is $\left(\begin{array}{cc}-2\left(q_{H}^{L H}-q_{L}^{L H}\right) & 0 \\ -2 q_{L}^{L H}\end{array}\right)$, which is negatively definite because of $q_{H}^{L H}>q_{L}^{L H}$. Solving the firstorder conditions $\partial \pi_{m}^{L H}\left(\theta_{1}, \theta_{2}, w_{A H}, w_{A L}\right) / \partial \theta_{1}=0$ and $\partial \pi_{m}^{L H}\left(\theta_{1}, \theta_{2}, w_{A H}, w_{A L}\right) / \partial \theta_{2}=0$ for $\left(\theta_{1}, \theta_{2}\right)$, we have

$$
\begin{gathered}
\theta_{1}^{L H}\left(w_{A L}, w_{A H}\right)=\frac{c_{B H}+q_{H}^{L H}-q_{L}^{L H}+w_{A H}-w_{A L}}{2\left(q_{H}^{L H}-q_{L}^{L H}\right)}, \\
\theta_{2}^{L H}\left(w_{A L}, w_{A H}\right)=\frac{q_{L}^{L H}+w_{A L}}{2 q_{L}^{L H}} .
\end{gathered}
$$

Inserting $\theta_{1}^{L H}$ and $\theta_{2}^{L H}$ into $D_{H}^{L H}, D_{L}^{L H}$, and $p_{H}^{L H}=q_{H}^{L H} \theta_{1}+$ $q_{L}^{L H}\left(\theta_{2}-\theta_{1}\right), p_{L}^{L H}=\theta_{2} q_{L}^{L H}$, we obtain the demands and retail prices of the manufacturer. Similarly, we can obtain the reaction functions when the manufacturer uses a common component.

Proof of Lemma 2. From $D_{H}^{L H}\left(w_{A L}, w_{A H}\right)>0$ and $D_{L}^{L H}\left(w_{A L}\right.$, $\left.w_{A H}\right)>0$, we have

$$
\left(q_{H}^{L H}-q_{L}^{L H}\right)-\left(w_{A H}-w_{A L}\right)>c_{B H}>\frac{q_{H}^{L H} w_{A L}}{q_{L}^{L H}}-w_{A H} .
$$

Similarly, we have the following:

$$
\begin{gathered}
q_{H}^{L L}-q_{L}^{L L}>c_{B H}>\frac{\left(q_{H}^{L L}-q_{L}^{L L}\right) w_{A L}}{q_{L}^{L L}} . \\
q_{H}^{H H}-q_{L}^{H H}>c_{B H}>\frac{\left(q_{H}^{H H}-q_{L}^{H H}\right) w_{A H}}{q_{L}^{H H}},
\end{gathered}
$$

By considering (A.2) and (A.3) together, we can have the unit wholesale prices intervals.
Proof of Lemma 3. Differentiating $\pi_{m}^{L H}\left(w_{A L}, w_{A H}\right)$ with respect to $w_{A H}$, we have

$$
\begin{aligned}
& \frac{\partial \pi_{m}^{L H}\left(w_{A L}, w_{A H}\right)}{\partial w_{A H}} \\
& =\frac{c_{B H}-q_{H}^{L H}+q_{L}^{L H}+w_{A H}-w_{A L}}{2\left(q_{H}^{L H}-q_{L}^{L H}\right)} \\
& =-D_{H}^{L H}\left(w_{A L}, w_{A H}\right)<0,
\end{aligned}
$$

and $\partial^{2} \pi_{m}^{L H}\left(w_{A L}, w_{A H}\right) / \partial w_{A H}^{2}=1 /\left[2\left(q_{H}^{L H}-q_{L}^{L H}\right)\right]>0$.

Similarly, using Lemma 2 and $q_{H}^{L L}-q_{L}^{L L}>c_{B H}$, we can show the other results.

Proof of Proposition 4. From $q_{L}^{L L}=q_{L}^{L H},(2)$, and (4), it follows that

$$
\begin{aligned}
F_{1}(\Delta w) & \\
=( & \left(q_{H}^{L L}-q_{L}^{L H}\right) \Delta w^{2}+2\left[c_{B H}-\left(q_{H}^{L H}-q_{L}^{L H}\right)\right] \\
& \cdot\left(q_{H}^{L L}-q_{L}^{L H}\right) \Delta w \\
& \left.+\left[\left(q_{H}^{L H}-q_{L}^{L H}\right)\left(q_{H}^{L L}-q_{L}^{L H}\right)-c_{B H}^{2}\right]\left(q_{H}^{L H}-q_{H}^{L L}\right)\right) \\
& \cdot\left(4\left(q_{H}^{L H}-q_{L}^{L H}\right)\left(q_{H}^{L L}-q_{L}^{L H}\right)\right)^{-1},
\end{aligned}
$$

where $\Delta w=w_{A H}-w_{A L}$. By differentiating $F_{1}(\Delta w)$ with respect to $\Delta w$ twice, we have $\partial^{2} F_{1}(\Delta w) / \partial \Delta w^{2}=1 /\left[2\left(q_{H}^{L H}-\right.\right.$ $\left.\left.2 q_{L}^{L H}\right)\right]>0$. Solving $F_{1}(\Delta w)=0$, we can obtain two positive solutions $\Delta w_{ \pm}$, where

$$
\begin{aligned}
\Delta w_{ \pm} & \\
= & \left(\left(q_{H}^{L H}-q_{L}^{L H}-c_{B H}\right)\left(q_{H}^{L L}-q_{L}^{L H}\right)\right. \\
& \left.\quad \pm\left(q_{H}^{L L}-q_{L}^{L H}-c_{B H}\right) \sqrt{\left(q_{H}^{L H}-q_{L}^{L H}\right)\left(q_{H}^{L L}-q_{L}^{L H}\right)}\right) \\
& \cdot\left(q_{H}^{L L}-q_{L}^{L H}\right)^{-1} .
\end{aligned}
$$

Solving the first-order condition $\partial F_{1}(\Delta w) / \partial \Delta w=0$ for $\Delta w$, we have $\Delta w_{1}=q_{H}^{L H}-q_{L}^{L H}-c_{B H}$. From Lemma 2, we know that $\Delta w<\Delta w_{1}$. So $F_{1}(\Delta w) \geq 0$ is equivalent to $\Delta w \leq \Delta w_{-}$.

Using $q_{H}^{H H}=q_{H}^{L H}$, we obtain $F_{20}=F_{2} \cdot 4 q_{L}^{L H} q_{L}^{H H}\left(q_{H}^{L H}-\right.$ $\left.q_{L}^{L H}\right)\left(q_{H}^{L H}-q_{L}^{H H}\right)$, where

$$
\begin{aligned}
F_{20}( & \left.w_{A L}, w_{A H}\right) \\
= & q_{L}^{H H} q_{H}^{L H}\left(q_{H}^{L H}-q_{L}^{H H}\right) w_{A L}^{2}-2\left(q_{H}^{L H}-q_{L}^{H H}\right) q_{L}^{L H} q_{L}^{H H} \\
& \cdot\left(c_{B H}+w_{A H}\right) w_{A L}+2 c_{B H} q_{L}^{L H}\left(q_{H}^{L H}-q_{L}^{H H}\right) \\
& \cdot q_{L}^{H H} w_{A H}+\left(q_{H}^{L H}-q_{L}^{H H}\right)\left(q_{L}^{L H}+q_{L}^{H H}-q_{H}^{L H}\right) q_{L}^{L H} w_{A H}^{2} \\
& +c_{B H}^{2} q_{L}^{L H}\left(q_{L}^{L H}-q_{L}^{H H}\right) q_{L}^{H H},
\end{aligned}
$$


which is a convex function of $w_{A L} \cdot F_{2} \geq 0$ is equivalent to $F_{20} \geq 0$. There are two solutions for $F_{20}\left(w_{A L}, w_{A H}\right)=0$, $w_{A L \pm}$. From $\partial F_{20}\left(w_{A L}, w_{A H}\right) / \partial w_{A L}=0$, we can have $w_{A L 1}=$ $q_{L}^{L H}\left(c_{B H}+w_{A H}\right) / q_{H}^{L H}$. From Lemma 2 , we see $w_{A L}<q_{L}^{L H}\left(c_{B H}+\right.$ $\left.w_{A H}\right) / q_{H}^{L H}$. Further, it follows that $F_{2}\left(w_{A L}, w_{A H}\right) \geq 0$ is equivalent to $w_{A L} \leq w_{A L_{-}}=A_{1} w_{A H}+B_{1}$. Similarly, from $q_{H}^{H H}-q_{L}^{H H}>c_{B H}, q_{H}^{H H}=q_{H}^{L L}-q_{L}^{L L}+q_{L}^{H H}$, and Lemma 2, it follows that $F_{3}\left(w_{A L}, w_{A H}\right) \geq 0$ is equivalent to $w_{A H} \leq w_{A H_{-}}$, where $w_{A H_{-}}$is given by Proposition 4 .

Using

$$
\begin{aligned}
q_{H}^{L H}-q_{H}^{L L} & =q_{L}^{H H}-q_{L}^{L H}, \\
q_{H}^{H H}-q_{L}^{H H} & =q_{H}^{L L}-q_{L}^{L L}, \\
q_{H}^{H H} & =q_{H}^{L H}, \\
q_{L}^{L L} & =q_{L}^{L H},
\end{aligned}
$$

we can find that $w_{A H}-w_{A L}=\Delta w_{-}, w_{A L}=w_{A L_{-}}$, and $w_{A H}=$ $w_{A H_{-}}$intersect in one point $\left(w_{A L 0}, w_{A H 0}\right)$. From $q_{H}^{L H}>q_{L}^{L H}$ and $q_{L}^{H H}>q_{L}^{L H}$, we see $A_{1}<1$. Now, we show that $1 / A_{1}>\sqrt{q_{L}^{H H} / q_{L}^{L H}}>1$, which is equivalent to $2 \sqrt{q_{L}^{H H} q_{L}^{L H}}+$ $2 \sqrt{\left(q_{H}^{L H}-q_{L}^{H H}\right)\left(q_{H}^{L H}-q_{L}^{L H}\right)}<2 q_{H}^{L H}$. This inequality is satisfied because of

$$
\begin{aligned}
2 \sqrt{q_{L}^{H H} q_{L}^{L H}} & \\
& +2 \sqrt{\left(q_{H}^{L H}-q_{L}^{H H}\right)\left(q_{H}^{L H}-q_{L}^{L H}\right)} \\
< & \left(q_{L}^{H H}+q_{L}^{L H}\right)+\left(q_{H}^{L H}-q_{L}^{H H}\right)+\left(q_{H}^{L H}-q_{L}^{L H}\right)=2 q_{H}^{L H} .
\end{aligned}
$$

Thus, we can differentiate the feasible regions for the unit wholesale prices into the three regions: I, II, and III. Further, we can complete the proof of Proposition 4.

Proof of Proposition 5. From (15), it follows that $\pi_{s}^{I}\left(w_{A L}, w_{A H}\right)$ is concave over $\left(w_{A L}, w_{A H}\right)$ because the Hessian matrix is negatively definite. Solving the first-order condition $\partial \pi_{s}^{I} / \partial w_{A L}=$ 0 and $\partial \pi_{s}^{I} / \partial w_{A H}=0$, we obtain $w_{A L 1}^{I}=\left(c_{A L}+q_{L}^{L H}\right) / 2$, $w_{A H 1}^{I}=\left(q_{H}^{L H}+c_{A H}-c_{B H}\right) / 2$ when we ignore the unit wholesale prices constraints.

Solving $w_{A H}=\left(w_{A L}-B_{1}\right) / A_{1}$ and $w_{A H}=w_{A L}+\Delta w_{-}$, we obtain $w_{A H 0}$ and $w_{A L 0}$, given by Proposition 5. From Proposition 4 , we know that only when $\left(w_{A L 1}^{I}, w_{A H 1}^{I}\right) \in I$, the manufacturer will buy two quality levels for component $A$. If $w_{A L 1}^{I}+\Delta w_{-} \geq w_{A H 1}^{I}$ and $w_{A H 1}^{I}<\left(w_{A L 1}^{I}-B_{1}\right) / A_{1}$, the supplier has equilibrium wholesale prices at the boundary $w_{A L}=$ $A_{1} w_{A H}+B_{1}$, because $F_{2}\left(w_{A L}, w_{A H}\right)$ decreases with $w_{A L}$. Inserting $w_{A L}=A_{1} w_{A H}+B_{1}$ into $\pi_{s}^{I}\left(w_{A L}, w_{A H}\right)$, we have $\pi_{s}^{I}\left(w_{A H}\right)$, which is a concave of $w_{A H}$. By solving the first-order condition, we have $w_{A H 2}^{I}$. If $w_{A H 2}^{I}<w_{A H 0}$, the unit wholesale prices are $\left(A_{1} w_{A H 2}^{I}+B_{1}, w_{A H 2}^{I}\right)$.

Similarly, we can obtain the other solutions; thus, in region $I$, the unit wholesale prices for the supplier are $\left(w_{A L}^{I *}, w_{A H}^{I *}\right)$ given by Proposition 5 .
From (16) and (17), it follows that $\pi_{s}^{I I}\left(w_{A L}\right)$ is a concave function of $w_{A L}$ while $\pi_{s}^{I I I}\left(w_{A H}\right)$ is a concave function of $w_{A H}$. So the optimal wholesale prices in the two regions are $w_{A L}^{I I *}=$ $\left(c_{A L}+q_{L}^{L L}\right) / 2$ and $w_{A H}^{I I *}=\left(c_{A H}+q_{L}^{H H}\right) / 2$. Furthermore, we can have

$$
\begin{aligned}
& \pi_{s}^{I I I}\left(w_{A H}^{I I *}\right)=\frac{\left(q_{L}^{H H}-c_{A H}\right)^{2}}{\left(8 q_{L}^{H H}\right)}, \\
& \pi_{s}^{I I}\left(w_{A L}^{I I *}\right)=\frac{\left(q_{L}^{L L}-c_{A L}\right)^{2}}{\left(8 q_{L}^{L L}\right)} .
\end{aligned}
$$

Proof of Corollary 6. Part (i) is obvious. Part (ii) is as follows: by substituting $w_{A L}^{I I *}$ and $w_{A H}^{I I I *}$ into $D_{L}^{L L}\left(w_{A L}\right)$ and $D_{L}^{H H}\left(w_{A H}\right)$, we can obtain $D_{L}^{H H *}=\left(2 c_{B H} q_{L}^{H H}-\left(q_{H}^{H H}-q_{L}^{H H}\right)\left(c_{A H}+\right.\right.$ $\left.\left.q_{L}^{H H}\right)\right) /\left[4 q_{L}^{H H}\left(q_{H}^{H H}-q_{L}^{H H}\right)\right]$ and $D_{L}^{L L *}=\left(2 c_{B H} q_{L}^{L L}-\left(q_{H}^{L L}-\right.\right.$ $\left.\left.q_{L}^{L L}\right)\left(c_{A L}+q_{L}^{L L}\right)\right) /\left[4 q_{L}^{L L}\left(q_{H}^{L L}-q_{L}^{L L}\right)\right]$. From $q_{H}^{H H}-q_{L}^{H H}=q_{H}^{L L}-q_{L}^{L L}$, it follows that $D_{L}^{H H *}<D_{L}^{L L *}$ is equivalent to $c_{A H} / c_{A L}>$ $q_{L}^{H H} / q_{L}^{L L}$. Using $D_{H}^{H H *}=D_{H}^{L L *}$, we can complete the proof. From (A.10), we can show part (iii).

Proof of Corollary 7. From Proposition 5, we can have that $w_{A L 1}^{I}+\Delta w_{-} \geq w_{A H 1}^{I}$ is equivalent to $\left(q_{H}^{L H}-q_{L}^{L H}-c_{B H}\right)-2 \Delta w_{-}+$ $c_{A H} \leq c_{A L}$, while $w_{A H 1}^{I} \geq\left(w_{A L 1}^{I}-B_{1}\right) / A_{1}$ is equal to $\left(c_{A H}+q_{H}^{L H}-c_{B H}\right) A_{1}+2 B_{1}-q_{L}^{L H} \geq c_{A L}$. So we have

$$
\begin{array}{r}
c_{A L} \in\left[\left(q_{H}^{L H}-q_{L}^{L H}-c_{B H}\right)-2 \Delta w_{-}\right. \\
\left.+c_{A H},\left(c_{A H}+q_{H}^{L H}-c_{B H}\right) A_{1}+2 B_{1}-q_{L}^{L H}\right] \\
\quad \text { if }\left(w_{A L 1}^{I}, w_{A H 1}^{I}\right) \in I .
\end{array}
$$

From Proposition 5, we see that the SPNE decisions under two scenarios are identical if the manufacturer uses common component $A$. We can complete the proof.

Proof of Proposition 8. Using $q_{L}^{L H}=q_{L}^{L L}$ and $q_{H}^{L H}=q_{H}^{H H}$, we obtain

$$
\begin{aligned}
\Delta \pi_{m 1} & \\
= & \frac{c_{B H}^{2}\left(4 q_{H}^{H H}-q_{H}^{L L}-3 q_{L}^{L L}\right)}{16\left(q_{H}^{L L}-q_{L}^{L L}\right)\left(q_{H}^{H H}-q_{L}^{L L}\right)} \\
+ & \left(\left(c_{A H}-c_{A L}\right)\left(2 q_{H}^{H H}-2 q_{L}^{L L}-c_{A H}+c_{A L}\right)\right. \\
& \left.\quad-2 c_{B H}\left(c_{A H}-c_{A L}+3 q_{H}^{H H}-3 q_{L}^{L L}\right)\right) \\
& \cdot\left(16\left(q_{H}^{H H}-q_{L}^{L L}\right)\right)^{-1} \\
+ & \frac{4 q_{H}^{L L}-q_{H}^{H H}-3 q_{L}^{L L}}{16}
\end{aligned}
$$


which is a concave function of $c_{A L}$ because of $q_{H}^{H H}>q_{L}^{L L}$. Note $q_{H}^{L L}-q_{L}^{L L}-c_{B H}>0$. Solving $\Delta \pi_{m 1}=0$ for $c_{A L}$, we have

$$
\begin{aligned}
c_{A L 1 \pm}= & c_{A H}+c_{B H}-q_{H}^{H H}+q_{L}^{L L} \\
& \pm 2\left(q_{H}^{L L}-q_{L}^{L L}-c_{B H}\right) \sqrt{\frac{\left(q_{H}^{H H}-q_{L}^{L L}\right)}{\left(q_{H}^{L L}-q_{L}^{L L}\right)}} .
\end{aligned}
$$

Thus, we see $\Delta \pi_{m 1} \geq 0$ for $c_{A L} \in\left[c_{A L 1-}, c_{A L 1+}\right]$.

Using $q_{L}^{L H}=q_{L}^{L L}$ and $q_{H}^{L H}=q_{H}^{H H}$, we obtain

$$
\Delta \pi_{m 2}=\frac{A_{2}+B_{2} c_{A L}-q_{H}^{H H} q_{L}^{H H}\left(q_{H}^{H H}-q_{L}^{H H}\right) c_{A L}^{2}}{16 q_{L}^{H H} q_{L}^{L L}\left(q_{H}^{H H}-q_{L}^{H H}\right)\left(q_{H}^{H H}-q_{L}^{L L}\right)},
$$

which is a concave function of $c_{A L}$ because of $q_{H}^{H H}>q_{L}^{H H}$. Solving $\Delta \pi_{m 2}=0$ for $c_{A L}$, we obtain

$$
c_{A L 2 \pm}=\frac{B_{2} \pm \sqrt{B_{2}^{2}+4 A_{2} q_{H}^{H H} q_{L}^{H H}\left(q_{H}^{H H}-q_{L}^{H H}\right)}}{2 q_{H}^{H H} q_{L}^{H H}\left(q_{H}^{H H}-q_{L}^{H H}\right)} .
$$

Thus, we have $\Delta \pi_{m 2} \geq 0$ when $c_{A L 2 \pm}$ exist and $c_{A L} \in$ $\left[c_{A L 2-}, c_{A L 2+}\right]$; otherwise, $\Delta \pi_{m 2}<0$.

Using $q_{H}^{H H}=q_{H}^{L L}-q_{L}^{L L}+q_{L}^{H H}$, we obtain

$$
\begin{aligned}
& \Delta \pi_{m 3} \\
& =\left(q_{L}^{L L}\left[c_{A H}^{2}-2 c_{A H} q_{L}^{H H}+q_{L}^{H H}\left(q_{L}^{H H}-q_{L}^{L L}\right)\right]\right. \\
& \left.\quad+2 q_{L}^{H H} q_{L}^{L L} c_{A L}-q_{L}^{H H} c_{A L}^{2}\right) \\
& \quad\left(16 q_{L}^{H H} q_{L}^{L L}\right)^{-1},
\end{aligned}
$$

which is an increasing and concave function of $c_{A L}$ because of $w_{A L 1}^{I}>c_{A L}$. Solving $\Delta \pi_{m 3}=0$ for $c_{A L}$, we obtain $c_{A L 3}=q_{L}^{L L}-$ $\left(q_{L}^{H H}-c_{A H}\right) \sqrt{q_{L}^{L L} / q_{L}^{H H}}$. Further, we see that $\Delta \pi_{m 3} \geq 0$ is equivalent to $c_{A L} \geq c_{A L 3}$.

\section{Conflict of Interests}

The authors declare that there is no conflict of interests regarding the publication of this paper.

\section{Acknowledgments}

This research was supported in part by (i) China National Funds for Distinguished Young Scientists under Grant 71425001 and (ii) the National Natural Science Foundation of China under Grant 71371093.

\section{References}

[1] P. Desai, S. Kekre, S. Radhakrishnan, and K. Srinivasan, "Product differentiation and commonality in design: balancing revenue and cost drivers," Management Science, vol. 47, no. 1, pp. 37-51, 2002.
[2] A. K. Chakravarty and N. Balakrishnan, "Achieving product variety through optimal choice of module variations," IIE Transactions, vol. 33, no. 7, pp. 587-598, 2001.

[3] U. W. Thonemann and M. L. Brandeau, "Optimal commonality in component design," Operations Research, vol. 58, no. 1, pp. $1-19,2000$.

[4] M. Fisher, K. Ramdas, and K. Ulrich, "Component sharing in the management of product variety: a study of automotive braking systems," Management Science, vol. 45, no. 3, pp. 297315, 1999 .

[5] F. Bernstein, A. G. Kök, and L. Xie, "The role of component commonality in product assortment decisions," Manufacturing \& Service Operations Management, vol. 13, no. 2, pp. 261-270, 2011.

[6] P. C. Verhoef, K. H. Pauwels, and M. A. Tuk, "Assessing consequences of component sharing across brands in the vertical product line in the automotive market," Journal of Product Innovation Management, vol. 29, no. 4, pp. 559-572, 2012.

[7] D. P. Rutenberg, "Design commonality to reduce multi-item inventory: optimal depth of a product line," Operations Research, vol. 19, no. 2, pp. 491-509, 1971.

[8] K. Kim, D. Chhajed, and Y. Liu, "Can commonality relieve cannibalization in product line design?" Marketing Science, vol. 32, no. 3, pp. 510-521, 2013.

[9] H. S. Heese and J. M. Swaminathan, "Product line design with component commonality and cost-reduction effort," Manufacturing \& Service Operations Management, vol. 8, no. 2, pp. 206$219,2006$.

[10] R. Subramanian, M. E. Ferguson, and L. Beril Toktay, "Remanufacturing and the component commonality decision," Production and Operations Management, vol. 22, no. 1, pp. 36-53, 2013.

[11] E. Labro, "The cost effects of component commonality: a literature review through a management-accounting lens," Manufacturing \& Service Operations Management, vol. 6, no. 4, pp. 358-367, 2004.

[12] S. K. Fixson, "Modularity and commonality research: past developments and future opportunities," Concurrent Engineering Research and Applications, vol. 15, no. 2, pp. 85-111, 2007.

[13] P. Lacourbe, "A model of product line design and introduction sequence with reservation utility," European Journal of Operational Research, vol. 220, no. 2, pp. 338-348, 2012.

[14] G. Van Ryzin and S. Mahajan, "On the relationship between inventory costs and variety benefits in retail assortments," Management Science, vol. 45, no. 11, pp. 1496-1509, 1999.

[15] U. W. Thonemann and J. R. Bradley, "The effect of product variety on supply-chain performance," European Journal of Operational Research, vol. 143, no. 3, pp. 548-569, 2002.

[16] T. Xiao and T. Xu, "Pricing and product line strategy in a supply chain with risk-averse players," International Journal of Production Economics, vol. 156, pp. 305-315, 2014.

[17] Y. C. Liu and T. H. Cui, "The length of product line in distribution channels," Marketing Science, vol. 29, no. 3, pp. 474-482, 2010.

[18] S. Rajagopalan and N. Xia, "Product variety, pricing and differentiation in a supply chain," European Journal of Operational Research, vol. 217, no. 1, pp. 84-93, 2012.

[19] T. Xiao, T. M. Choi, and T. C. Cheng, "Product variety and channel structure strategy for a retailer-Stackelberg supply chain," European Journal of Operational Research, vol. 233, no. 1, pp. 114-124, 2014. 
[20] M. Mussa and S. Rosen, "Monopoly and product quality," Journal of Economic Theory, vol. 18, no. 2, pp. 301-317, 1978.

[21] K. S. Moorthy, "Market segmentation, self-selection, and product line design," Marketing Science, vol. 3, no. 4, pp. 288-307, 1984.

[22] J. M. Villas-Boas, "Product line design for a distribution channel," Marketing Science, vol. 17, no. 2, pp. 156-169, 1998.

[23] V. Krishnan and W. Zhu, "Designing a family of developmentintensive products," Management Science, vol. 52, no. 6, pp. 813825, 2006.

[24] S. Netessine and T. A. Taylor, "Product line design and production technology," Marketing Science, vol. 26, no. 1, pp. 101-117, 2007.

[25] S. Chayet, P. Kouvelis, and D. Z. Yu, "Product variety and capacity investments in congested production systems," Manufacturing \& Service Operations Management, vol. 13, no. 3, pp. 390-403, 2011.

[26] D. Z. Yu, "Product variety and vertical differentiation in a batch production system," International Journal of Production Economics, vol. 138, no. 2, pp. 314-328, 2012.

[27] T. Xiao and X. Qi, "Strategic wholesale pricing in a supply chain with a potential entrant," European Journal of Operational Research, vol. 202, no. 2, pp. 444-455, 2010.

[28] Y. Liu and R. K. Tyagi, "The benefits of competitive upward channel decentralization," Management Science, vol. 57, no. 4, pp. 741-751, 2011.

[29] K. Kim and D. Chhajed, "Commonality in product design: cost saving, valuation change and cannibalization," European Journal of Operational Research, vol. 125, no. 3, pp. 602-621, 2000. 


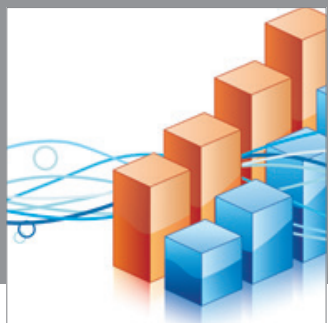

Advances in

Operations Research

mansans

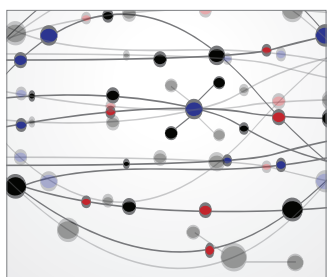

The Scientific World Journal
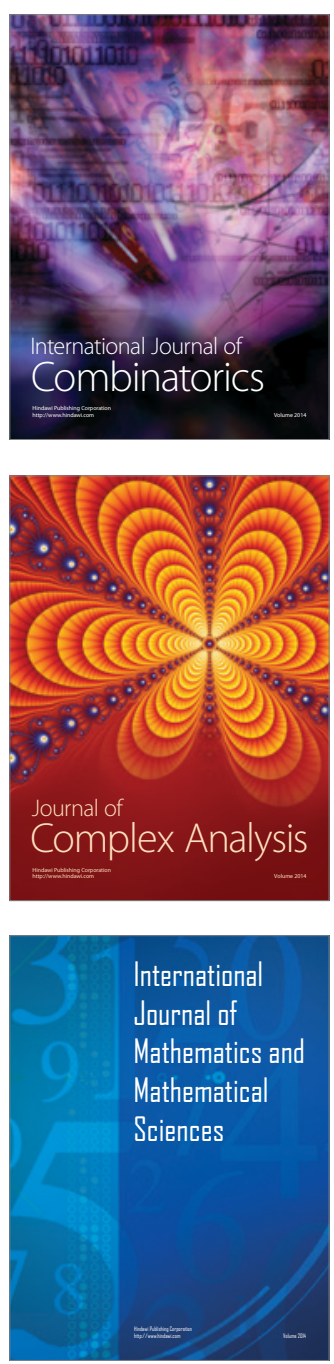
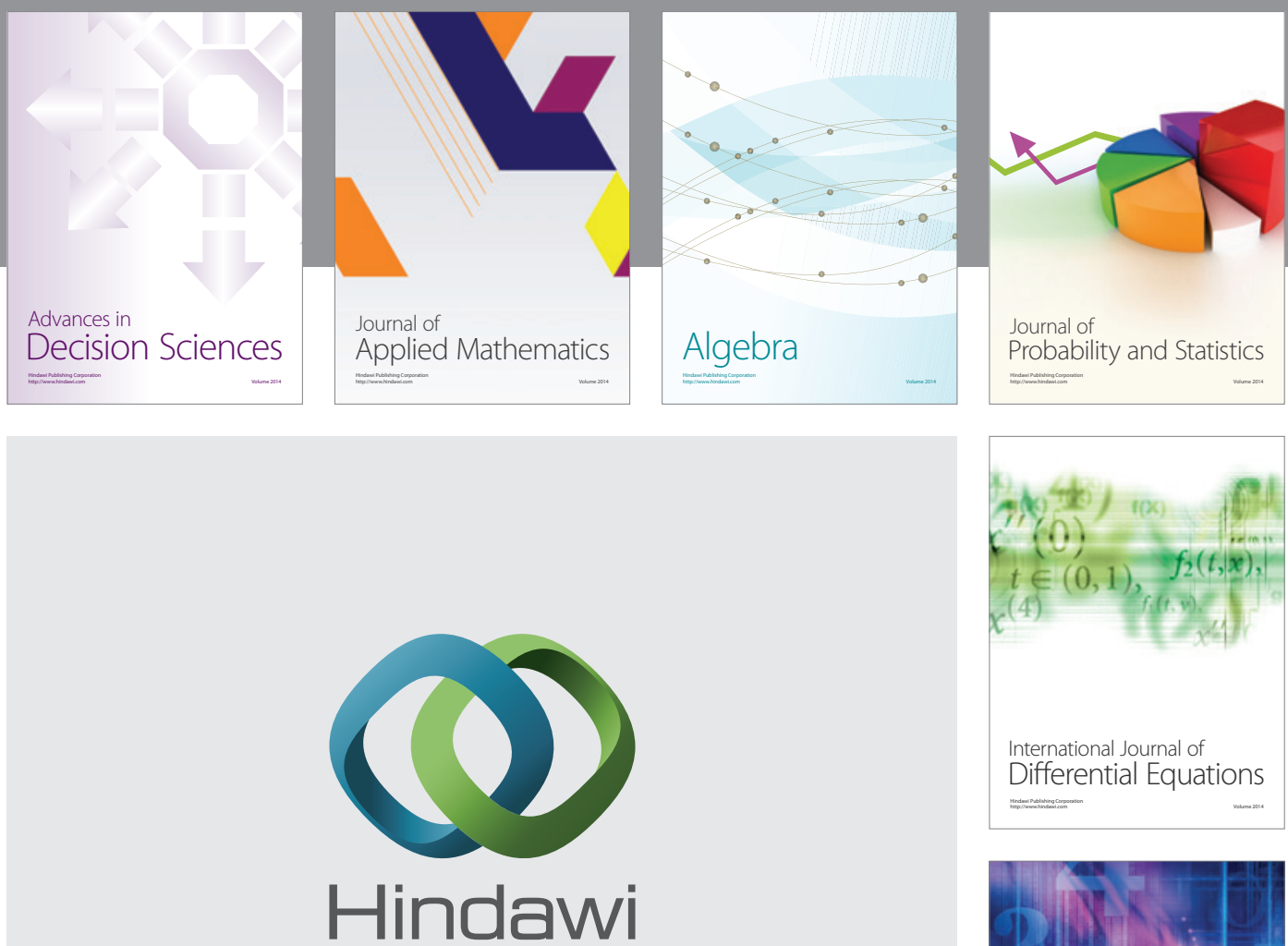

Submit your manuscripts at http://www.hindawi.com
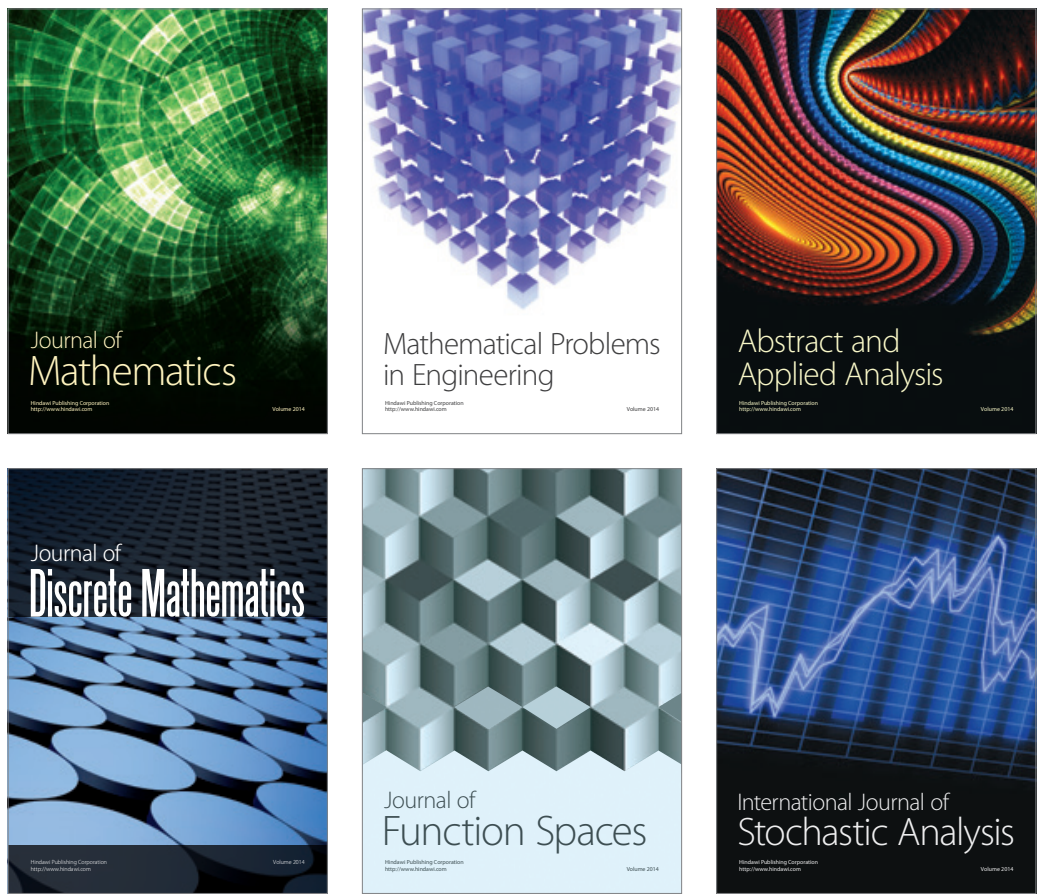

Journal of

Function Spaces

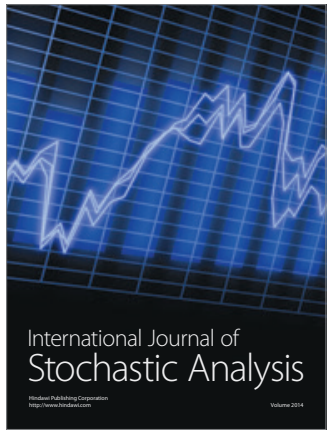

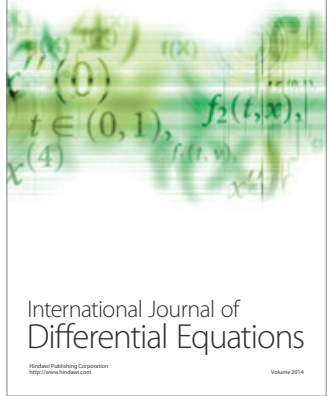
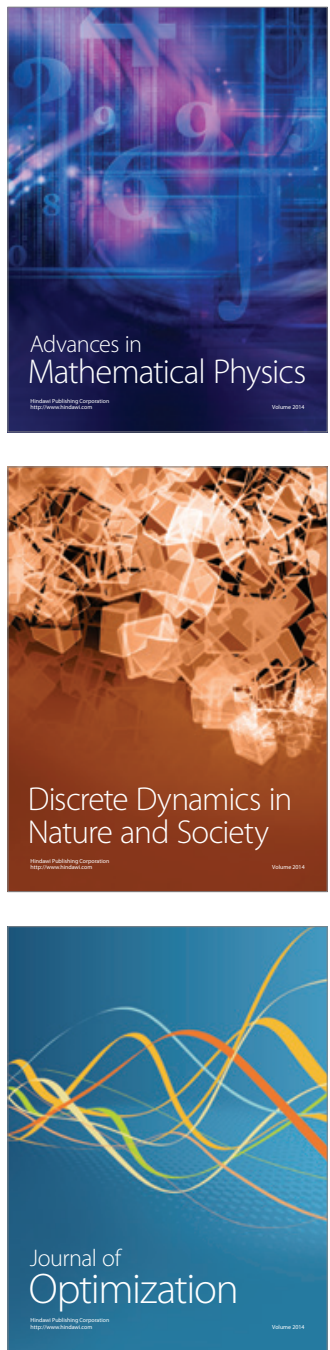